\title{
An MRI Investigation of Kimura Disease (KD) in Upper Extremity Soft Tissues: Two Case Reports
}

\author{
Renwei Liu ${ }^{1}$, Yindi Zhang ${ }^{2}$, Jinzhi Fang ${ }^{1}$ and Yuzhong Zhang ${ }^{1, *}$ \\ ${ }^{1}$ Department of Radiology, People's Hospital of Longhua District, Shenzhen, China \\ ${ }^{2}$ Department of Radiology, the First Affiliated Hospital of Kunming Medical University, Kun Ming Yunnan, China \\ "Corresponding author: Yuzhong Zhang, Department of Radiology, People's Hospital of Longhua District, No. 2 Construction of East Road, Shenzhen, 518109, China. Tel: \\ +861-8898834345, E-mail: 1090237421@qq.com
}

Received 2017 July 24; Revised 2017 November 13; Accepted 2017 December 09.

\begin{abstract}
Kimura disease (KD) is a rare chronic granulomatous inflammation disease with unknown causes, which is common in young Asian males. Local lymph node drainages are characteristic of the disease, the most common of which occur in the salivary glands. Other reported drainages occur in the axilla, popliteal fossa, groin, and upper extremities. Unfortunately, there not many characteristic manifestations that typically present in patients with KD. Sometimes only increased peripheral blood eosinophils and serum IgE concentration are the only positive presentations. Therefore, it is very difficult for physicians to diagnose KD. We report herein on two cases of KD. Both of them presented in the soft tissues of the upper extremities. Special attention was given to the MRI findings of the two patients with a view to disclosing specific characteristics that should help in the realization of clinical diagnosis.
\end{abstract}

Keywords: Kimura Disease, Magnetic Resonance Imaging, Upper Extremities

\section{Background}

MRI has a high resolution for soft tissues. Preoperative MRI examination is an excellent tool for observation of the internal structure, blood supply, and the relationship between a lesion, its nerves, and the surrounding and internal vessels. It is also useful in the differentiation of Kimura disease (KD) and other soft tissue tumors, such as mesenchymal sarcomas. This report summarizes an investigation into two cases of KD with reference to related literature, with a view on evaluating the value of MRI in the prognosis of KD.

\section{Case Presentation}

The study was approved by the ethical committee of the People's hospital of Longhua district, China. Written consent was obtained.

\subsection{MRI Protocol}

MRI examinations were performed with a 1.5-Tesla MRI system (Achieva, Philips healthcare, Amsterdam, Netherlands) with a soft coil. Axial, coronal, and sagittal planes T1WI-turbo spin echo (TSE) (fast spin echo sequences, repetition time (TR) 488/Ef, echo time (TE) 15/Ef), field of view (FOV): $18 \times 18$, number of excitations (NEX) $=4$, axial, coronal, and sagittal planes T2WI-TSE (fast spin echo sequences, TR3627/Ef, TE100/Ef), FOV: $20 \times 20$, NEX = 4. The two patients underwent enhanced scans, a. Axial, coronal and sagittal planes T1WI-TSE-spectral presaturation with inversion recovery (SPIR)+contrast enhanced (CE) (TR500/Ef, TE15/Ef), FOV:20 × 20, NEX = 3. Gadoliniumdiethylenetriamine pentaacetic acid (Gd-DTPA) dosage was $0.2 \mathrm{mmol} / \mathrm{kg}$, the flow rate was $3.0 \mathrm{~mL} / \mathrm{s}$.

\subsection{Case 1}

A 24-year-old male had a left upper extremity mass excision five years ago that recurred one year ago. The patient had no complaints or symptoms. Physical exams showed a soft firm mass, approximately $5.0 \mathrm{~cm} \times 4.0 \mathrm{~cm}$ $\times 4.0 \mathrm{~cm}$ in size. Blood analysis revealed a peripheral blood eosinophil percentage of 35.3\%, (standard: 0.4 - 0.8\%) and an eosinophil absolute value of $3.05 \times 10^{9} / \mathrm{L}$ (standard: $\left.0.02-0.52 \times 10^{9} / \mathrm{L}\right)$. Ultrasound examination revealed a non-uniform mixed lesion with rich blood flow. Magnetic resonance images showed a lesion with slightly homogeneous hyper intense signals on both the T1- and T2weighted images (Figure $1 \mathrm{~A}$ and $1 \mathrm{~B}$ ), the contrast enhancement format was heterogeneous (Figure 1C). The patient underwent a mass excision procedure. The pathology suggested Kimura disease (Figure 1D). 

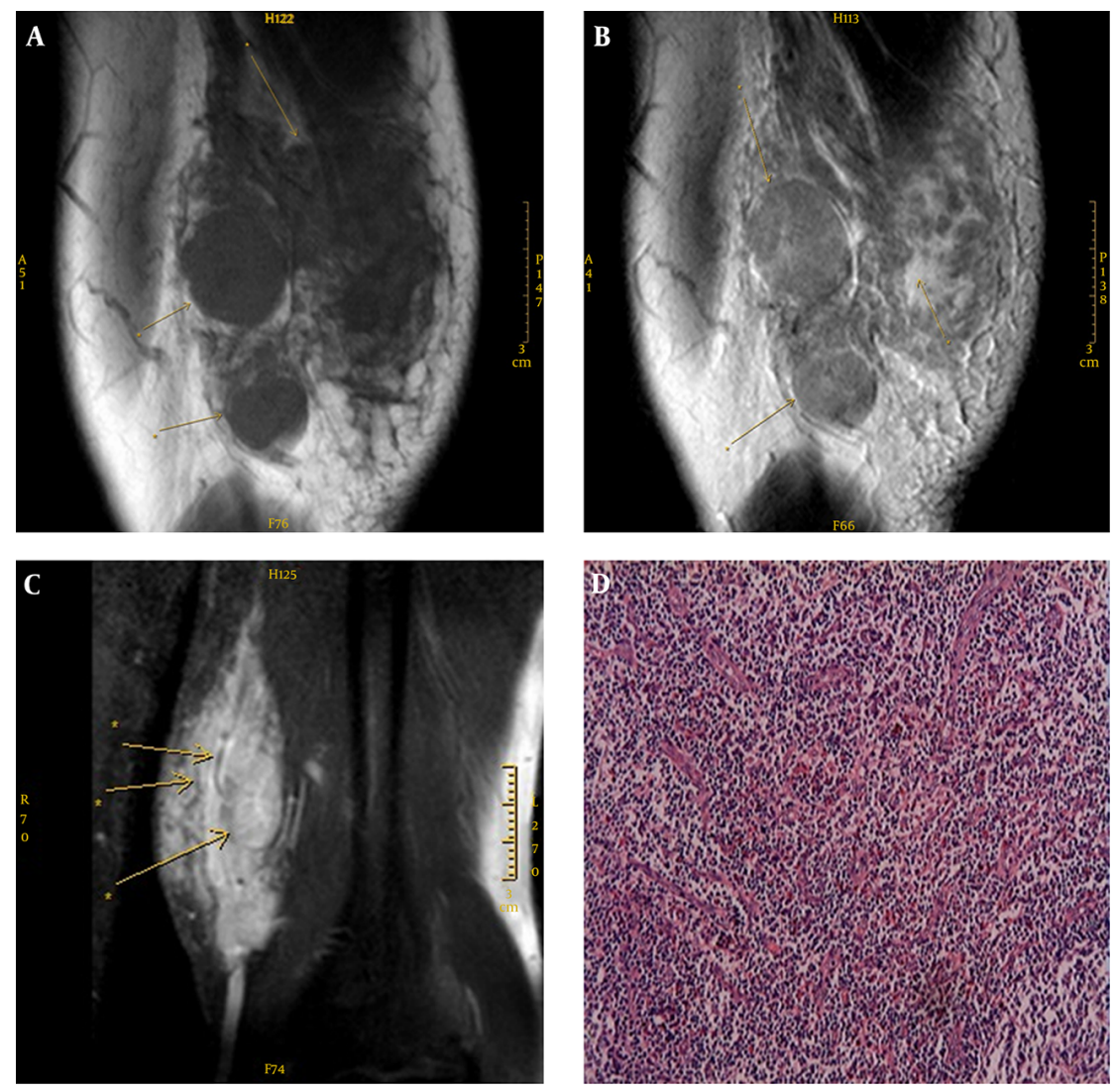

Figure 1. Kimura disease of the left upper extremity in a 24 year-old Chinese man (case 1). A, In sagittal T1-weighted imaging, the lesion shows slightly increased signal intensity compared to normal muscle; B, In sagittal T2-weighted image, the lesion shows high intensity; C, In contrast-enhanced T1-weighted image, heterogeneous enhancement is demonstrated with some nodular structures; some flow voids, representing vessels, can also be identified; D, Histopathology of the mass and lymph nodes revealed dense infiltration of eosinophils, lymphocytes, histiocytes with suffused small vessels, and mild fibrosis (H \& E stain, $\times 20$ ).

\subsection{Case 2}

A 39 year-old male presented with a painless soft mass in the right upper extremity and reported no other complaints or symptoms. A soft firm mass, approximately $7.0 \mathrm{~cm} \times 3.0 \mathrm{~cm} \times 3.0 \mathrm{~cm}$ in size was found upon physical examination. Blood analysis revealed a peripheral eosinophil percentage of $26.1 \%$ and an eosinophil absolute value of $2.23 \times 10^{9} / \mathrm{L}$, the serum IgE level was 1700 $\mathrm{U} / \mathrm{mL}$. Ultrasound examination showed a heterogeneous lesion. Magnetic resonance images revealed the lesion was slightly hyper intense in the T1-weighted images (Figure $2 \mathrm{~A}$ ) and hyper intense in the T2-weighted images (Figure 2B). Enhancement was also found in this patient (Figure 2C). MRI images also disclosed the mass had multi-nodule formation. The mass surrounded the right brachial vein, and the smaller nodule size was approximately $1.5 \mathrm{~cm} \times 1.8$ $\mathrm{cm} \times 1.8 \mathrm{~cm}$. The patient underwent a mass excision procedure. The pathology suggested Kimura disease (Figure 2D).

\section{Discussion}

Eosinophilic lymphogranuloma, also known as Kimura disease (KD) is reported to occur over a large age range, from 5 to 80 years, and with a male to female ratio of up to 10:1 (1). Most studies on the radiological characteristics of KD patients report on its manifestation in the head and neck (2), and rarely in the upper arm, as was the focus in the two cases studied in this case report $(3,4)$.

Choi et al. (4) reported on nine cases of upper arm $\mathrm{KD}$, with a focus on the MRI characteristics. All of the masses showed signal intensity similar or slightly higher than the muscle in the T1-weighted images and hyperintensity signals similar to muscle in the T2-weighted images. In gadolinium-enhanced T1-weighted images, homogeneous enhancement in the lesion was observed in seven cases. In this study, the T1-weighted images from the two cases exhibited slightly hyper intense signals. T2-weighted images showed hyper intense signals, which were signifi- 

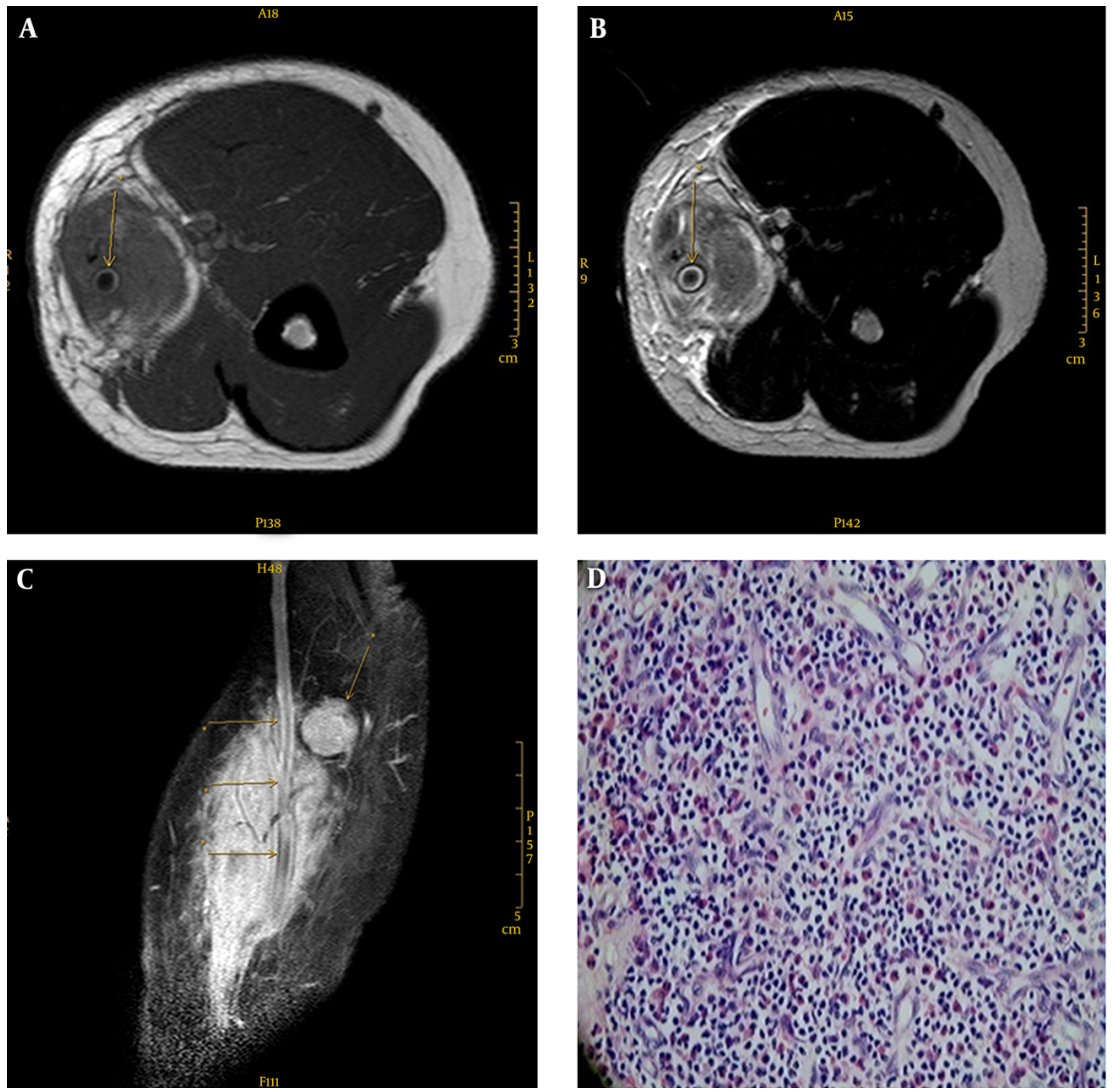

Figure 2. Kimura disease of the right upper extremity in a 39 year-old Chinese man (case 2). A, In axial T1-weighted image, the subcutaneous lesion shows a clear boundary and slightly elevated increased signal; B, In axial T2-weighted image, the lesion shows a highly increased signal, the basilic vein is engulfed but normal in size and without signs of thrombosis; C, In sagittal T1-weighted fat suppression image after Gd-administration, obvious enhancement can be observed; D, Histopathology of the mass and lymph nodes reveals dense infiltration of eosinophils, lymphocytes, histiocytes with diffuse small vessels, and mild fibrosis $(\mathrm{H} \& \mathrm{E}$ stain, $\times 20)$.

cantly enhanced but not uniform, with internal linear or tubular visible enhancement (most likely proliferation of the capillary shadow) and a few cable-like non-enhanced scattered regions (probably formation of fibrous tissue). Gopinathan et al. (5) classified KD into two distinct types; type 1 is well-defined with a nodular configuration; type 2 is ill-defined with a plaque-like configuration. Grossly, the two cases reported in this report were both grape-like and without capsules. The lesion could be divided into two parts. One was solid tissue, located in the center of the lesion, and the other was composed of round nodules surrounding the lesion. Strips and patchy fibers were visible between these two regions. Some small vessels were also identified in the center of the lesions. We believe that a "third type" should be added to KD identification, a mixed type, with characteristics typical of both type 1 and type 2 , as observed in both cases studied here. Additionally, the MRI scans also detailed the blood vessel wall edges and signals clearly.
In conclusion, MRI findings of KD in the upper extremity include specific locations along the neuro-lymphovascular structures, the absence of necrosis or calcification, multiple flow voids representing vascular structures, and various amounts of edema in the adjacent subcutaneous fat. Neurovascular structures were without signs of direct invasion. The lesions show slightly hyper intense signals on axial T1-weighted images and hyper intense signals on axial T2-weighted images, compared to normal muscle. The diagnosis of KD should consider combining with elevated peripheral blood eosinophilia and serum IgE levels.

\section{Footnotes}

Authors' Contributions: All authors have participated equally.

Funding/Support: None.

Conflict of Interests: None. 


\section{References}

1. Takeishi M, Makino Y, Nishioka H, Miyawaki T, Kurihara K. Kimura disease: diagnostic imaging findings and surgical treatment. JCraniofac Surg. 2007;18(5):1062-7. doi: 10.1097/scs.0b013e3180f61249. [PubMed: 17912083].

2. Li J, Zhang R, Liu X, Mo Y, Lv Y, Xie C. CT and MRI findings of Kimura disease. Chinese J Radiol. 2010;44(6):619-22.

3. Sanders M, Raj R, Miller M, Clark M. Kimura's disease: upper limb involvement in a Pacific Island man. ANZ J Surg. 2003;73(6):465-7.
[PubMed: 12801352].

4. Choi JA, Lee GK, Kong KY, Hong SH, Suh JS, Ahn JM, et al. Imaging findings of Kimura's disease in the soft tissue of the upper extremity. AJR Am J Roentgenol. 2005;184(1):193-9. doi: 10.2214/ajr.184.1.01840193. [PubMed: 15615973].

5. Gopinathan A, Tan TY. Kimura's disease: imaging patterns on computed tomography. Clin Radiol. 2009;64(10):994-9. doi: 10.1016/j.crad.2009.07.003. [PubMed: 19748005]. 